\title{
Tracing radioactive contamination of river basins for the development of effective mitigation measures
}

\author{
Chihiro Yoshimura $^{1} \cdot$ Takashi Gomi $^{2} \cdot$ Kazumi Tanida $^{3}$ \\ Published online: 6 January 2018 \\ (c) International Consortium of Landscape and Ecological Engineering and Springer Japan KK, part of Springer Nature 2018
}

\section{Foreword}

The accident at the Fukushima Daiichi Nuclear Power Plant in March 2011 resulted in the release of several hundred petabecquerels of radioactive elements into the environment. These radioactive elements were dispersed and deposited in the wide region of central Honshu Island, Japan, and atmospheric and hydrological processes carried considerable fractions even farther around the globe. In the 6 years since the accident, considerable effort has been made to reveal the fate and transport of these radioactive elements in major ecosystems, leading to significant advancement in our understanding of the relevant natural processes, as well as the effects of radioactivity at different levels in ecosystems.

We are honored to include several excellent papers (five original research articles and one short communication) on this in this special issue of Landscape and Ecological Engineering "Tracing radioactive contamination of river basins for the development of effective mitigation measures." Through this issue, we aim to provide updated scientific information on biogeochemical processes of radioactive substances for the effective management of river basin systems under radioactive stresses. Listed below, the articles in this issue comprehensively describe the fate and transport of radioactive cesium mostly in forested river basins. Key research findings compiled here provide us with a framework to explain where the radioactive cesium was dispersed and where it accumulated in forested rivers, how aquatic

Chihiro Yoshimura

yoshimura.c.aa@m.titech.ac.jp

1 Department of Civil and Environmental Engineering, Tokyo Institute of Technology, Tokyo, Japan

2 Department of International Environmental and Agricultural Science, Tokyo University of Agriculture and Technology, Tokyo, Japan

3 Osaka Museum of Natural History, Osaka, Japan invertebrates and vertebrates stored and metabolized the radioactive cesium in their bodies/tissues, and how radioactive cesium contaminated riparian food webs in forested rivers. In addition, the findings in this special issue substantially enhance our fundamental understanding of ecological processes in forested streams as radioactive cesium serves as an environmental tracer.

We are grateful to all the authors, as well as the anonymous reviewers, who worked on this special issue; without their great efforts, we could not have produced it. We also would like to thank the International Exchange Committee of the Ecology and Civil Engineering Society of Japan, as it kindly provided us with this opportunity to make this substantial scientific contribution.

\section{Papers in the special issue}

1. Characteristics of radiocesium contamination of dry riverbeds due to the Fukushima Daiichi Nuclear Power Plant accident assessed by airborne radiation monitoring. Kazuhiro Azami, Takahiro Ootagaki, Mutsushi Ishida, Yukihisa Sanada.

2. Evaluating cesium-137 detachment from coniferous needle litter in a headwater stream: a litter bag field experiment. Takashi Gomi, Masaru Sakai, M. Enamul Haque, Kosuke Hosoda, Hiroto Toda.

3. "Caddisfly watch," a biomonitoring program using Stenopsyche larvae to determine radioactive cesium contamination in rivers following the Fukushima nuclear disaster. Daisuke Ueno, Hazuki Mizukawa, Osamu Inanami, Hiromitsu Nagasaka, Nozomi Tatsuta, Yukinori Narazaki, Takeshi Fujino, Izumi Watanabe, Yutaka Kameda, Kunihiko Nakai.

4. Radioactive cesium contamination and its biological half-life in larvae of Stenopsyche marmorata (Trichoptera: Stenopsychidae). Takeshi Fujino, Shota Kobori, Takeshi Nomoto, Masaru Sakai, Takashi Gomi. 
5. Seasonal variation in food web-based transfer factors of radiocesium in white-spotted char Salvelinus leucomaenis from headwater streams. M. Enamul Haque, Takashi Gomi, Masaru Sakai, Junjiro N. Negishi.

6. Cesium-137 contamination of river food webs in a gradient of initial fallout deposition in Fukushima, Japan.
Junjiro Negishi, Masaru Sakai, Kengo Okada, Aimu Iwamoto, Takashi Gomi, Kazuki Miura, Masanori Nunokawa, Mitsuru Ohira. 